\title{
EFFECT OF SIMVASTATIN ON IODOACETATE-INDUCED TEMPORO- MANDIBULAR JOINT OSTEOARTHRITIS IN RATS
}

\author{
Abdelrahman Mohamed Galal*, Said Mahmoud Hani**, Ahmed Mohamed Soliman***
}

\begin{abstract}
Objectives: Osteoarthritis (OA) is one of the harmful disorders affecting the body joint including the temporomandibular joint (TMJ). Simvastatin is presumed to possess antiosteoarthritic effect beside its antilipidemic influences, so that the present study tried to estimate its effect on the monoiodoacetate (MIA)-induced TMJOA. Subjects and Methods: thirty young adult female Sprague-Dawley rats were randomly categorized into; control, osteoarthritis and osteoarthritis with simvastatin groups. Both MIA for $\mathrm{OA}$ induction and simvastatin for therapy were delivered unilaterally via the intra-articular route at the right side as a single dose for each. Results: the simvastatin administration has relatively reduced the MIA-induced osteoarthritic manifestations, and/or inhibited the progress of some osteoarthritic degenerative features but it failed to control others. Conclusion: temporomandibular joint osteoarthritis (TMJOA) treatment by simvastatin showed relative antiosteoarthritic effect and modulated the structure of some joint components to compensate the damaged induced structures with MIA administration.
\end{abstract}

KEYWORDS: iodoacetate, OARSI score, osteoarthritis, simvastatin, temporomandibular joint

\section{INTRODUCTION}

The TMJ is a unique joint allowing for articulation between the mandibular condyle and the mandibular fossa of the temporal bone. The fibrocartilage of the mandibular condyle is comprised of extracellular matrix (ECM) proteins and proteoglycans that provide the necessary properties to resist the forces elaborated on the condyle and mandibular fossa during mastication and joint maturation ${ }^{(1)}$. The TMJ disorders afflict approximately $5-10 \%$ of the US population ${ }^{(2)}$. These disorders encompass collection of at least 12 clinical phenotypes, one of which is the degenerative TMJOA ${ }^{(3)}$. The individuals with this degenerative joint disease experience irreversible damage to the joint complex, including the degradation and loss of the extracellular matrix (ECM) proteins necessary for the joint function. Unfortunately, in most cases, this insidious and irreversible damage occurs before the later development of the symptoms and the diagnosis of the disease ${ }^{(4)}$.

Several reports of the age-related changes in articular cartilage are consistent with the phenotypes of chondrocyte senescence. These age-related changes are manifested with telomere shortening and changes in cell morphology, cellular viability and matrix protein production, suggested that the chondrocyte aging occurs even with the donor aging $(5,6)$.

The MIA is a metabolic inhibitor that destroys the joint cartilage by blocking the glyceraldehyde3-phosphate dehydrogenase and glycolysis in chondrocytes. The rapid depletion of adenosine triphosphate (ATP) due to inhibition of glycolysis

\footnotetext{
* PhD Candidate, Oral and Dental Biology Department, Faculty of Dentistry, Al-Azhar University, Boys, Cairo, Egypt ** Professor of Oral and Dental, Biology Department, Faculty of Dental Medicine, Al-Azhar University, Cairo, Egypt *** Lecturer of Oral and Dental Biology Department, Faculty of Dental Medicine, Al-Azhar University, Cairo, Egypt
} 
ultimately results in cellular death. The MIA administration has been used in different species, including mices and rats in order to experimentally induce $\mathrm{OA}^{(7,8)}$.

Statins are best known as the competitive inhibitors of hydroxymethyl-glutaryl- coenzyme-A reductase that can reduce the cholesterol biosynthesis. Moreover, it has been recognized that these agents also have significant antiinflammatory and immunomodulating effects ${ }^{(9-11)}$. The statin has been also shown to increase the mRNA levels of bone morphogenetic protein-2, aggrecan and collagen type II beside increasing the proteoglycan synthesis in rat chondrocytes ${ }^{(12)}$. Furthermore, the statins can reduce the chondrocyte senescence ${ }^{(13)}$. These findings suggest a predictable mechanism for the statin in counteracting TMJOA involving cartilage degeneration that encouraged the performance of the present study.

The OA Research Society International (OARSI) recently introduced the OARSI Osteoarthritis Cartilage Histopathology Assessment System (OOCHAS). The OARSI scoring system assesses cartilage pathogenesis by measuring both the vertical (grade) and horizontal (stage) progression of OA cartilage manifestations. The overall score is defined as the combined OA grade $0-6$ points and OA stage 0-4 points, representing a combined assessment of OA severity and extent $0-24$ points $^{(14)}$.

\section{SUBJECTS AND METHODS}

Thirty young adult $(180 \pm 50 \mathrm{~g})$ female Sprague Dawley rats were housed in the animal research unit, Cairo University. The experiment was performed according to Ethical Research Committee Protocol of Dental Medicine Faculty, Al-Azhar University. The procedures were conducted in accordance with Committee for the Purpose of Control and Supervision on Experiments on Animals (CPCSEA Guidelines).

Three groups of animals were randomly selected, including: Group (I); 10 rats represented the negative control for the osteoarthritic group. Group
(II); 10 rats were subjected to TMJOA induction by MIA. The TMJOA induction was committed by a single dose of $1 \mathrm{mg}$ Sodium MIA (Sigma ${ }^{\mathrm{TM}}$, St. Louis, MO, USA) dissolved in $50 \mu \mathrm{L}$ of distilled water in the right side via intraarticular route. Animals were sacrificed one week after TMJOA induction. Group (III): 10 rats were treated, one week after OA induction, with single dose of

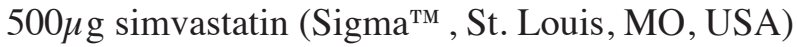
dissolved in $50 \mu \mathrm{L}$ of distilled water via intraarticular route ${ }^{(15)}$. The animals were sacrificed, four weeks after simvastatin administration.

After the end of experimental period, the rats were sacrificed by decapitation. The skulls were sagittally divided into two halves and the right side was soaked for 72 hours in $10 \%$ neutral buffered formalin for fixation. The hemicrania were demineralized and paraffinized and the paraffin embedded TMJ blocs were sagittally cut, in serial sections at 5-7 $\mu \mathrm{m}$ thickness, so that the tissue sections were parallel to the lateral surface of the mandibular ramus.

The tissue sections of TMJ in all groups of animals were microtechnically processed for histological investigations comprising; hematoxylin and eosin $(H \& E)$ stain, and, Toluidine Blue (TB) stain in order to survey (a) structural changes induced by the different injected materials and (b) perform OA score in the various experimental groups according to OARSI scoring system. The OA scoring was estimated according to two parameters; the grade and stage in accordance to the grade and stage scale proposed by Pritzker KPH, et al $2006^{(14)}$. The final score was defined as assessment of combined OA grade and OA stage. The data were recorded, and statistically analyzed, by SPSS 20 software, in which the P-values were given for comparison of the mean difference between control group and experimental groups using the independent sample t-test. One-way analysis of variance (ANOVA) test was used for comparison between different groups of animals. 


\section{RESULTS}

\section{Control group (G I)}

Histologically: the joint components showed intact and typical configuration; as the mandibular condyle was composed of typical structure from the articular surface inward; including the condylar fibrous layer, condylar proliferative layer, condylar cartilage layer and condylar subarticular bone containing large bone marrow spaces with interlacing numerous bone trabeculae. The roof of glenoid fossa was lined with fibrous tissue layer composed of densely arranged collagen fibers overlaying compact bone containing few, oval bone marrow spaces with some few bone marrow tissues.

The articular disc was composed of dense collagenous fibrous tissue containing densely arranged coarse collagen bundles with some interspersed flattened fibroblast. The synovium was composed of; cellular intima with one to four layers of synovial cells embedded into fiber free matrix and vascular subintima composed of loose connective tissue layer containing variably sized vascular elements (Fig. 1). The toluidine blue stain revealed intense metachromatic stain through the matrix of the condylar fibrous, proliferative, and cartilaginous layers (Fig. 2). The OARSI score was zero indicating the absence any OA features (Diagram 1).

\section{Effects of MIA-induced osteoarthritis on TMJ (G II)}

Histologically; the mandibular condyle demonstrated thinning or uneven thickness of both the fibrous and cartilaginous layers with occasional absence of condylar fibrous layer and the condylar proliferative layer. The chondrocytes irregularly arranged and orientated with some cells were degenerated, while the existing cells appeared atypical. The condylar subarticular bone undergone moderate sclerosis as reflected by the transformation of spongy bone into cortical bone with reduction or even disappearance of bone marrow spaces. The condylar subarticular bone marrow cavities were lined with cuboidal osteoblasts with occasional absence of these cells along the cavity surface, whereas the typical bone marrow was replaced with dense fibrous tissue.
The articular disc was reduced in thickness, while its peripheral parts, where it joins the articular capsule, showed fraying and disorientation of its constitutional collagen fibers with the development of interfibrillar edema (Figs. 3). The toluidine blue stain revealed light to moderate metachromasia throughout the matrix of all layers of mandibular condyle. Some areas, however, demonstrated fading out of metachromasia that became restricted to narrow rings surrounding the irregularly arranged and widely distributed chondrocytes (Fig. 4). Accordingly, the OARSI score was 12 that is the highest among the studied groups (Diagram 1).

\section{Effects of simvastatin on MIA-induced TMJOA (G III)}

Histologically: the simvastatin administration into the osteoarthritic TMJ evoked different modulation in the different joint compartments. The most striking finding, noted in most animals, was the marked increase in the thickness of condylar proliferative layer due to its profuse cell proliferation. The condylar fibrous layer was paramountly thickened posteriorly and was highly cellular in some animals, while others showed absence of the condylar fibrous layer. The hyperplastic condylar proliferative layer showed massive multiplication of randomly arranged prechondrocytes.

Osteophyte formation was developed in the anterior region of mandibular condyle as a prominence extending from the anterior pole of condyle interlocking with the anterior band of the articular disc. The fibrocartilaginous osteophyte was composed of chondroid bone surrounded by proliferative layer with haphazardly arranged collagen fibers and fibroblasts. The condylar subarticular bone appeared sclerotic in direct contact with the condylar proliferative layer (Figs. 5). The toluidine blue stain was intensely metachromatic in the matrix of condylar cartilage layer, particularly in the lower two thirds, while the metachromasia in the upper one third was lighter or even absent (Fig. 6). These findings were mirrored in the OARSI score, which recorded a lower level than that recorded in G II. 


\section{Photos captions abbreviations}

TFL: temporal fibrous layer - TB: temporal bone

TDS: temporodiscal space - AD: articular disc

CDS: condylodiscal space - CFL: condylar fibrous layer

CPL: condylar proliferative layer

CCL: condylar cartilage layer BT: bone trabeculae

CSB: condylar subarticular bone -BM: bone marrow

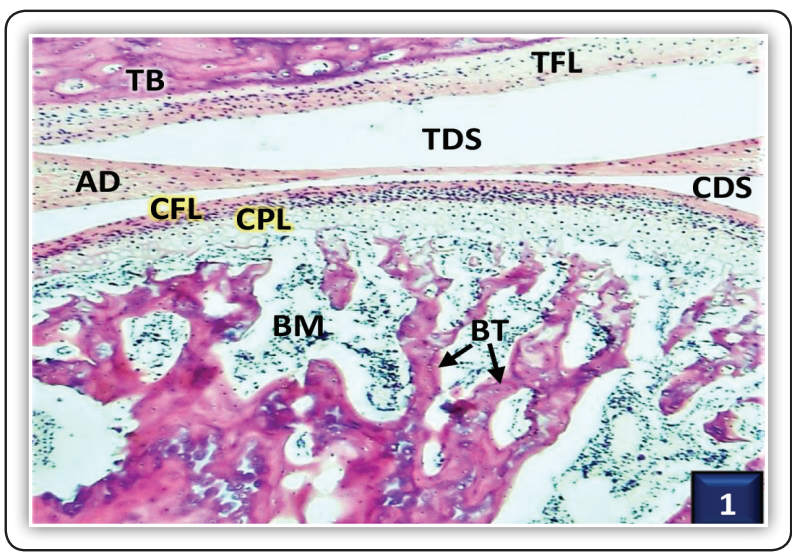

FIG (1) Sagittal sections of rat TMJ of control group (G I) showing the main structural components and their positional relations to each other (H\& E stain, Orig. Mag. X 40)

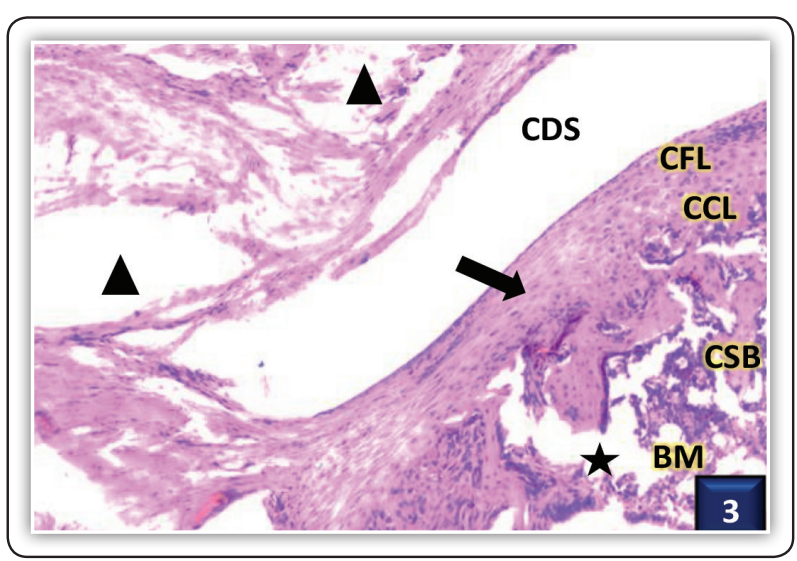

FIG (3) Sagittal sections of rat TMJ of osteoarthritis group (G II) showing regional absence of CCL with occasional abnormal proportion between CFL and CCL. The AD, where it joins articular capsule, shows fraying with development of interfibrillar edema (arrowheads). The CSB trabeculae appear randomly arranged, few, sclerotic and invaded by cracks filled by fibrotic bone marrow and edema (star). (H \& E stain, Orig. mag. X 100).

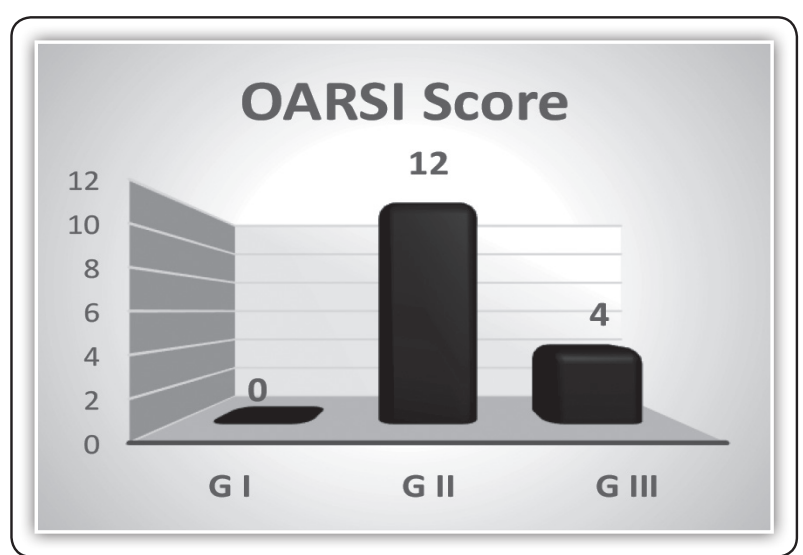

Diagram (1): the OARSI score

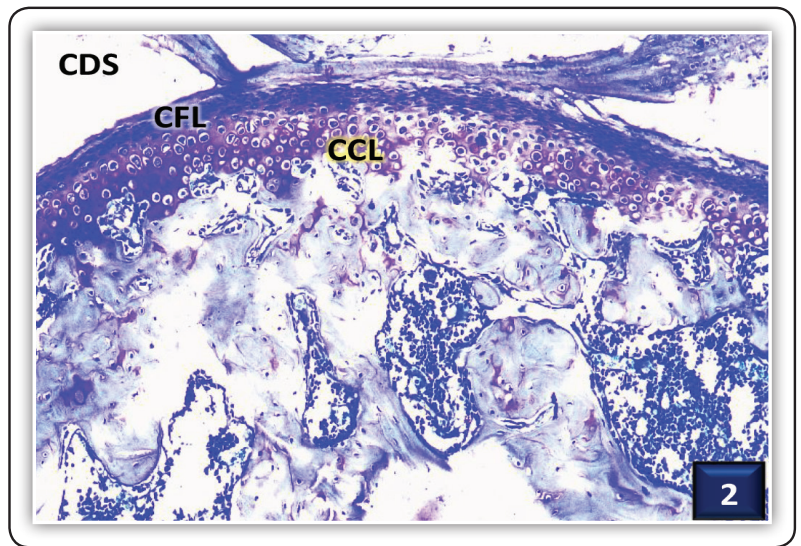

FIG (2) Sagittal sections of rat TMJ of control group (G I) showing intense reaction throughout the condylar cartilage in the matrix and the rims around chondrocytes. (TB stain. Orig. Mag. X 100)

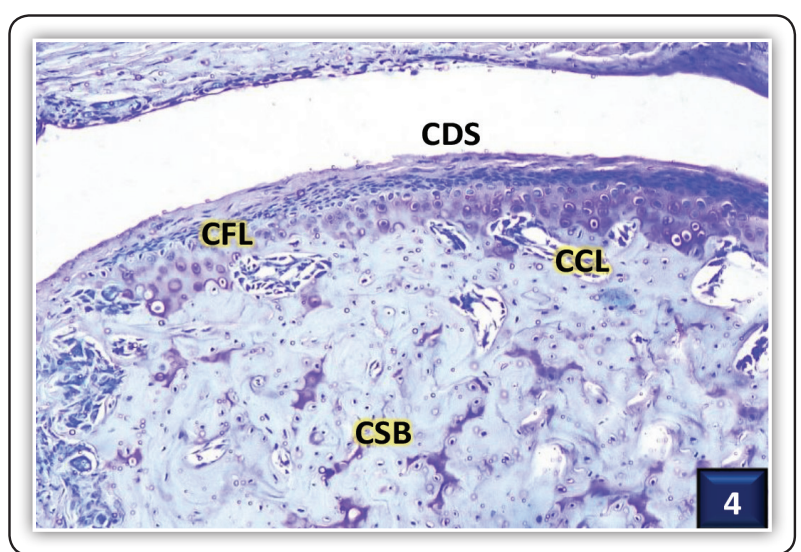

FIG (4) Sagittal sections of rat TMJ of osteoarthritis group (G II) showing metachromatic staining of CFL and CCL with moderate stain around some chondrocytes in CCL (right) but intense stain where different constituting layers of mandibular condyle are still preserved (left). (TB stain. Orig. Mag. X 100). 


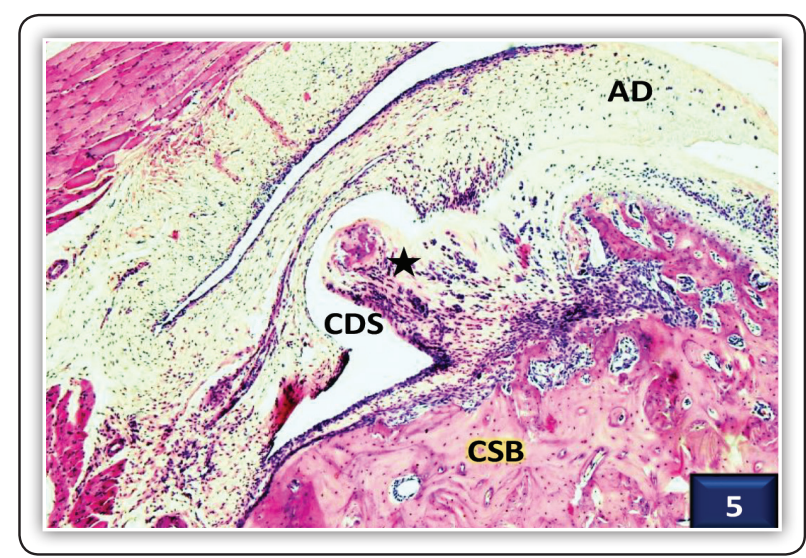

FIG (5) Sagittal sections of rat TMJ of osteoarthritis and simvastatin group (G III) showing osteophyte formation (star) at the anterior pole of the. The CSB appears highly sclerotic while the several superficial bone marrow spaces contain fibrous tissue and are evacuated into CPL. The AD becomes thickened at its anterior part. (H \& E stain, Orig. Mag. X 40).

\section{DISCUSSION}

Following the administration of MIA, the TMJ demonstrated many degenerative osteoarthritic changes involving all the joint components, particularly concentrated in the mandibular condyle. The MIA-induced TMJOA changes were reflected by the condylar chondrocytes degeneration and this phenomenon may be induced by apoptosis which could be an important initiator for the cartilage degeneration. The findings of the present investigation may be consistent with the previous studies, in respect to the most affected component of TMJ in the osteoarthritic group that is the condylar cartilage, the chondrocytes in particular. The imbalance between the anabolic and catabolic processes in the extracellular matrix simulates an already proved mechanism of osteoarthritis pathogenesis that has been stated in the previous investigations ${ }^{(16)}$. In a rat model of MIA-induced TMJOA, the apoptosis of chondrocytes is the prominent characteristic feature of the early phase of cartilage degradation, and the cytokines released by the apoptotic cartilage chondrocytes may contribute to the destruction of subchondral bone that ultimately caused abnormal bone remodeling ${ }^{(17)}$.

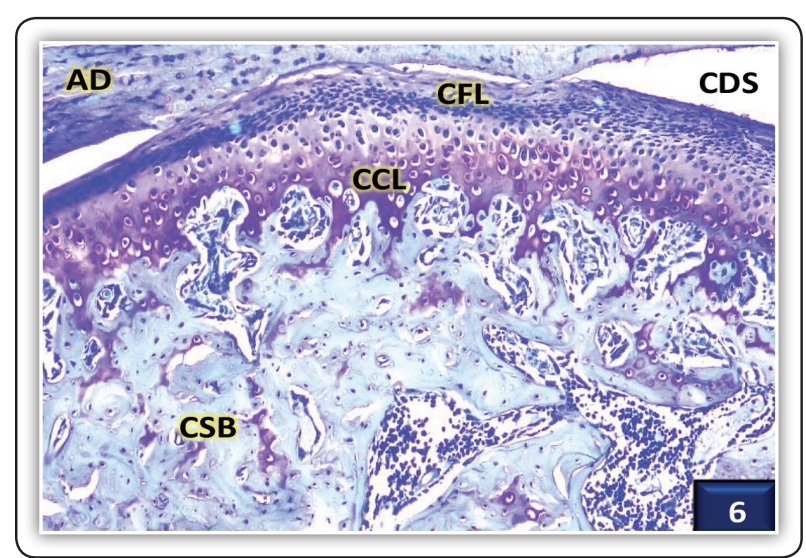

FIG (4) Sagittal sections of rat TMJ of osteoarthritis and simvastatin group (G III) showing intense stain in the CFL and moderate stain in upper layer of CCL, but intense stain in its lower part. The CSB appears faintly stained. (T.B. stain, Orig. Mag. X 100)

The results of the present study showed that statin have relative chondroprotective action that represented by the proliferation of chondrocytes precursors leading to the prominent thickening of condylar proliferative layer. Alternatively, the enhanced chondrocytes proliferation noted by simvastatin administration recorded in the present study, could be attributed to the previously reported statin ability to inhibit the senescence of articular chondrocytes ${ }^{(18)}$.

This anti-senescence potential of statin was recorded in a previous study that subjected the chondrocytes to the catabolic factor IL- $1 \beta$ which is commonly upregulated in osteoarthritis. In addition, the statin was believed to inhibit the IL$1 \beta$-induced shortening of the lifespan of cultured chondrocytes $^{(18)}$.

The simvastatin antiinflammatory potentials were reflected in its ability to prevent catabolic stress-induced downregulation of chondrocytes activity and to hinder the accelerated cellular aging in articular cartilage ${ }^{(18)}$. It seems that the previously reported anti-senescence ability of statin was represented, in the present study, by the marked 
chondrocytes proliferation. Moreover, the antiinflammatory action of simvastatin was reflected, in the present study, in the noticeable fall in the OARSI score compared to the score in the osteoarthritic group.

In contrast to previous investigations, the present study revealed that simvastatin administration not only failed to prevent the progression of some osteoarthritic features including the condylar subarticular bone sclerosis but also it promoted the osteophyte development. The osteoinductive effect, via the activation of osteoblastic activity, of statin could interpret the development of osteophyte at the margin of the mandibular condyle as well as the persistence of condylar subarticular bone sclerosis ${ }^{(11)}$. However, the OARSI score revealed that simvastatin administration had relatively reduced the OA severity, but not restored the TMJ back to normal.

\section{CONCLUSION}

The present investigation showed many degenerative features rather than inflammatory ones following MIA administration into the TMJ of rat. On the other hand, the simvastatin demonstrated abortive chondroreparative attempts though it promoted prominent chondrocytes precursors proliferation, but the proliferating cells did not arrange into the typical well-organized cartilage layers. The OARSI score showed lowered OA severity with simvastatin administration, however, the simvastatin disadvantageous effects were its failure to manage some of osteoarthritic manifestations and the enhanced osteophyte development that may be attributed to its osteo-inductive potential. Indeed, further studies are needed to overcome the simvastatin drawbacks and potentiate its beneficial effects.

\section{REFERENCES}

1. Shirakura M, Kram V, Robinson J, Sikka S, Kilts TM, Wadhwa S, et al. Extracellular Matrix Mediates BMP-2 in a Model of Temporomandibular Joint Osteoarthritis. Cells Tissues Organs, 2017; 204 (2):84-92.
2. Liu, F, Steinkeler A. Epidemiology, diagnosis, and treatment of temporomandibular disorders. Dent Clin North Am, 2013; 57: 465-79

3. Schiffman, E, Ohrbach R, Truelove E. Diagnostic criteria for temporomandibular disorders (DC/TMD) for clinical and research applications: recommendations of the International RDC/TMD Consortium Network and Orofacial Pain Special Interest Group. J Oral Facial Pain Headache, 2014; 28: 6-27.

4. Wang XD, Zhang JN, Gan YH, Zhou YH. Current understanding of pathogenesis and treatment of TMJ osteoarthritis. J Dent Res, 2015; 94: 666-73.

5. Guerne PA, Blanco F, Kaelin A, Desgeorges A, Lotz M. Growth factor responsiveness of human articular chondrocytes in aging and development. Arthritis Rheum, 1995; 38:960-8.

6. Martin JA, Buckwalter JA. Aging, articular cartilage chondrocyte senescence and osteoarthritis. Biogerontology, 2002; 3: 257-64.

7. Chandran P, Pai M, Blomme EA, Hsieh GC, Decker MW, Honore P. Pharmacological modulation of movementevoked pain in a rat model of osteoarthritis. Eur J Pharmacol, 2009;613(1-3):39-45.

8. Hadipour-Jahromy M, Mozaffari-Kermani R. Chondroprotective effects of pomegranate juice on monoiodoacetate-induced osteoarthritis of the knee joint of mice. Phytother Res, 2010;24(2):182-5.

9. Abeles AM, Pillinger MH. Statins as antiinflammatory and immunomodulatory agents: a future in rheumatologic therapy. Arthritis Rheum, 2006;54:393-407.

10. Baker JF, Walsh P, Mulhall KJ. Statins: a potential role in the management of osteoarthritis. Joint Bone Spine, 2011;78:31-34.

11. Lazzerini PE, Capecchi PL, Selvi E. Statins and the joint: multiple targets for a global protection. Semin Arthritis Rheum, 2011;40:430-46.

12. Hatano H, Maruo A, Bolander ME, Sarkar G. Statin stimulates bone morphogenetic protein-2, aggrecan, and type 2 collagen gene expression and proteoglycan synthesis in rat chondrocytes. J Orthop Sci, 2003; 8:842-8.

13. Conaghan PG. The effects of statins on osteoarthritis structural progression: another glimpse of the Holy Grail. Ann Rheum Dis, 2012;71(5):633-4.

14. Pritzker KPH, Gay S, Jimenez SA, Ostergaard K, Pelletier JP, Revell PA, Salter D, et al. Osteoarthritis cartilage his- 
topathology: grading and staging. Osteoarthritis and Cartilage, 2006; 14, 13-29.

15. Holwegner C, Reinhardt AL, Schmid MJ, Marx DB, Reinhardt RA. Impact of local steroid or statin treatment of experimental temporomandibular joint arthritis on bone growth in young rats. Am J Orthod Dentofacial Orthop, 2015; $147: 80-8$.

16. Wang XD, Kou XX, He DQ, Zeng MM, Meng Z, Bi RY, et al. Progression of cartilage degradation, bone resorption and pain in rat temporomandibular joint osteoarthritis induced by injection of iodoacetate. PLoS One, 2012; 7(9): e45036.

17. Suvarna SK. Layton C, Bancroft JD. Bancroft's theory and practice of histological techniques, Eighth edt, China, Elsevier Ltd, 2019: 176- 97.

18. Yudoh K, Karasawa R. Statin prevents chondrocyte aging and degeneration of articular cartilage in osteoarthritis (OA). Aging, 2010; 2 (12):990-8. 